\title{
Retraction Note: Induced-charge electrokinetic phenomena
}

\author{
Yasaman Daghighi · Dongqing Li
}

Published online: 21 July 2012

(C) Springer-Verlag 2012

Retraction to: Microfluid Nanofluid (2010) 9:593-611

DOI 10.1007/s10404-010-0607-2

The article has been retracted by request of the authors. Unaltered text was taken from a pre-published version of Bazant MZ, Squires TM (2010) Induced-charge electrokinetic phenomena. Curr Opin Colloid Interface Sci 15(2010) 203-213. Moreover, a few reproduced figures from other published articles lack appropriate references. The authors apologize for their negligence.

The online version of the original article can be found under doi:10.1007/s10404-010-0607-2.

Y. Daghighi · D. Li $(\bowtie)$

Department of Mechanical and Mechatronics Engineering, University of Waterloo, Waterloo, ON N2L 3G1, Canada

e-mail: dongqing@mme.uwaterloo.ca 